Unfallchirurg $2022 \cdot 125: 723-730$

https://doi.org/10.1007/s00113-021-01072-w

Angenommen: 20. Juli 2021

Online publiziert: 31. August 2021

(c) Der/die Autor(en) 2021

Redaktion

Wolf Mutschler, München

Hans Polzer, München

Ben Ockert, München

\section{Ambulantisierungspotenzial stationärer Fälle einer universitären Klinik für Orthopädie und Unfallchirurgie}

\author{
Jeanette Henkelmann ${ }^{1} \cdot$ Ralf Henkelmann ${ }^{2} \cdot$ Nikolaus von Dercks $^{3}$ \\ ${ }^{1}$ Klinik und Poliklinik für Diagnostische und Interventionelle Radiologie, Universitätsklinikum Leipzig AöR, \\ Leipzig, Deutschland \\ ${ }^{2}$ Klinik und Poliklinik für Orthopädie, Unfallchirurgie und Plastische Chirurgie, Universitätsklinikum \\ Leipzig AöR, Leipzig, Deutschland \\ ${ }^{3}$ Stabstelle Medizincontrolling, Universitätsklinikum Leipzig AöR, Leipzig, Deutschland
}

Hintergrund: Durch das Reformgesetz des Medizinischen Dienstes der Kassen (MDK) soll u. a. eine Verlagerung bislang stationär erbrachter Leistungen in den ambulanten Versorgungsektor bzw. die Versorgung nach §115b SGB V umgesetzt werden. Ziel dieser Arbeit ist die Untersuchung bestimmter Gruppen stationärer Fälle eines universitären Maximalversorgers für Unfallchirurgie und Orthopädie, die das Risiko einer operativen Ambulantisierung tragen.

Methodik: Die Datenerfassung mittels SAP Data Warehouse umfasst alle stationären Fälle 2017-2019. Es erfolgt die Subgruppenanalyse der Krankenhausleistungsparameter von 3 potenziellen Risikogruppen (RG): 1) primäre Fehlbelegungen, 2) Katalogleistungen der AOP-Kategorie 1 und/oder 2 sowie 3) elektive Eintagesfälle als hypothetische Risikogruppe. Zudem erfolgt eine Analyse epidemiologischer und ökonomischer Parameter.

Ergebnisse: Eine primäre Fehlbelegung (RG 1) wurde vom MDK in 245 Fällen beanstandet. RG 2 umfasst 764 Fälle und RG 3891 Fälle. Das Kollektiv wies ein Durchschnittsalter von 45,5 $\pm 17,7$ Jahren auf und zeigte in $90 \%$ keine relevanten Nebendiagnosen (PCCL 0). Der Hauptanteil der Fälle ließ sich den DRG I23B und I21Z (Entfernung von Osteosynthesematerial, 15-23\%) zuordnen, nachfolgend offenen oder arthroskopischen Eingriffen an den Extremitäten (DRG I32F, I32G, I24Z, I18B, 6-9\%). Im Falle einer zunehmenden Ambulantisierung ergibt sich ein potenzielles Erlösrisiko 2017 von 1.049.207 €, 2018 von $1.076 .727 €$ und 2019 von $923.163 €$. Schlussfolgerung: Einzelne Gruppen haben ein erhöhtes Transferpotenzial in bestimmten DRG für ambulante Operationen. Eine proaktive Patientensteuerung in Bezug auf ambulante vs. stationäre Behandlung sowie ein besonderes Management personeller und räumlicher Ressourcen sind notwendig, um nachgelagerte Erlöskürzungen zu antizipieren.

\section{Schlüsselwörter}

Ambulantes Operieren · DRG · Kodierung · MDK-Prüfung $\cdot$ Abrechnung

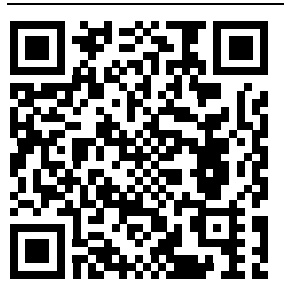

QR-Code scannen \& Beitrag online lesen
Die Rangfolge „ambulant vor stationär” folgt dem Wirtschaftlichkeitsgebot unseres Gesundheitssystems. Das MDKReformgesetz fordert nun eine weitere Verlagerung bislang stationär erbrachter Leistungen in den ambulanten Versorgungsektor nach § 115b SGB V [6]. Durch eine geplante Erweiterung des Ka- talogs für ambulante Operationen und stationäre Behandlungen soll das Ambulantisierungspotenzial gefördert und gleichzeitig dem Entstehen eines der häufigsten Überprüfungsgründe entgegengewirkt werden. 


\section{Hintergrund und Fragestellung}

Der medizinische Fortschritt ermöglicht die Durchführung vieler Operationen ambulant mindestens in gleicher, wenn nicht sogar in überlegener Qualität und zu geringeren Kosten als stationär $[1,2,2$, 15-18, 20, 23]. Vorteile können medizinisch wissenschaftlich verortet sein, z. B. in Form einer geringeren Exposition gegenüber oft problematischen nosokomialen Krankheitserregern. Zudem entspricht die ambulante Durchführung dem Patientenbedürfnis, einhergehend mit einer geringeren psychischen Belastung $[3,10]$.

Mit dem Gesetz für bessere und unabhängigere Prüfungen des Medizinischen Dienstes der Krankenversicherung (MDKReformgesetz) soll u.a. das ambulante Potenzial auf der Grundlage der aktuellen medizinischen Erkenntnisse neu definiert werden [8]. Die Rangfolge "ambulant vor stationär" folgt dem Wirtschaftlichkeitsgebot gemäß § 12 sowie dem § 39 SGB V $[5,7]$. Demnach sollen bestehende ambulante Behandlungsmöglichkeiten in Krankenhäusern durch Erweiterung des Katalogs für ambulante Operationen und stationäre Ersatzverfahren ausgeweitet und gleichzeitig dem Entstehen eines der häufigsten Überprüfungsgründe seitens der Kostenträger entgegengewirkt werden. Nach § 115b Absatz 1a SGB V hätte durch den Spitzenverband Bund der Krankenkassen, die Deutsche Krankenhausgesellschaft und die kassenärztlichen Bundesvereinigungen bis zum 30.06.2020 ein gemeinsames Gutachten beauftragt werden sollen, in dem der Stand der medizinischen Erkenntnisse zu ambulant durchführbaren Operationen, stationsersetzenden Eingriffen und Behandlungen (AOP-Katalog) untersucht wird [8]. Veranlasst durch die COVID-19-Pandemie und auf Grundlage des Zweiten Gesetzes zum Schutz der Bevölkerung bei einer epidemischen Lage von nationaler Tragweite wurden die Implementierung und Anwendung des überarbeiteten AOP-Katalogs auf das Jahr 2022 verschoben [9].

Als Ziel dieser Arbeit soll das Ambulantisierungsrisiko stationärer Fälle der Klinik und Poliklinik für Orthopädie, Unfallchirurgie und Plastische Chirurgie (KOUP) des Universitätsklinikums Leipzig untersucht werden. Für einen Transfer potenziell am- bulant behandelbarer Patienten sollen entsprechende Risikogruppen herausgearbeitet und ökonomisch bewertet werden.

\section{Methodik}

\section{Datenerhebung}

Die Datengrundlage umfasst alle entlassenen stationären Fälle der Jahre 2017, 2018 und 2019 der KOUP, welche sich auf die 5 fachlichen Organisationseinheiten Unfallchirurgie (UCh), Arthroskopie/ gelenkerhaltende Chirurgie (ACh), plastische und Handchirurgie (PCh), Orthopädie (ORT) und Wirbelsäulenchirurgie (WCh) verteilen. Die Erfassung der Falldaten erfolgte mittels SAP Data Warehouse (Fa. SAP Deutschland, Walldorf) hinsichtlich epidemiologischer Merkmale (Alter, Geschlecht) sowie der Leistungsparameter (Hauptdiagnose (ICD-10-GM), Verweildauer, patientenbezogener klinischer Schweregrad $[P C C L])$. Der $P C C L$ als kumulativer Schweregrad von Komplikationen und Komorbiditäten der einzelnen Diagnosen wird in den Stufenwerten 0 bis 6 durch den DRG-Grouper ausgegeben.

\section{Risikogruppen}

Aus allen Fällen wurden 3 Risikogruppen gebildet:

- Gruppe 1 (primäre Fehlbelegungen): Hierfür erfolgte die Erfassung der Behandlungsfälle mit primärer Fehlbelegung nach MDK-Prüfung der Jahre 2017-2019. Diese Gruppe hat keine Schnittmenge mit Gruppe 2 und 3, da die Fälle nach der Prüfung nicht mehr als "stationär" im SAP-Datenbestand geführt werden.

- Gruppe 2 (AOP-Katalog Kategorie 1 und/oder 2): Es wurden die Behandlungsfälle erfasst, deren operativer Eingriff entsprechend dem OPS-Kode ausschließlich einer Kategorie 1 und/ oder 2 des AOP-Katalogs zugeordnet werden kann. Fälle mit zusätzlichen Prozeduren ohne Zuordnung zu einer dieser Kategorien wurden ausgeschlossen.

- Gruppe 3 (elektive Eintagesfälle): Hierunter wurden alle Behandlungsfälle mit folgenden Merkmalen erfasst: Alter $\geq 18$ Jahre, Verweildauer ein Tag,
Beatmungsstunden $=0$, DRG-Partition: operativ, $\mathrm{PCCL}=0$, Aufnahmeart: (normale) Einweisung, Entlassart: (normale) Entlassung.

Die Risikogruppen 2 und 3 entstammen einer gemeinsamen Datengrundlage und weisen eine Schnittmenge auf. Um eine doppelte ökonomische Betrachtung zu vermeiden, wird die Schnittmenge gesondert erfasst.

Die potenziellen Erlöse aus dem ambulanten Operieren wurden als Produkt aus dem mittleren Erlös des ambulanten Operierens des jeweiligen Jahres mit der Fallzahl an Risikofällen des gleichen Jahres ermittelt.

\section{Datenanalyse}

Es werden gängige Größen wie Fallzahl, Case-Mix (CM, Summe der Bewertungsrelationen aller Fälle) und Case-Mix-Index (CMI, Summe der Bewertungsrelationen, dividiert durch die Fallzahl [durchschnittliche Bewertungsrelation pro Fall]), berücksichtigt. Der Landesbasisfallwert (LBFW) beträgt für Sachsen 2017 3278,19€, 2018 $3341,67 €$ und für $20193528,65 €$. Es sollen eine epidemiologische und ökonomische Bewertung der Behandlungsfälle der einzelnen Risikogruppen und eine fiktive Erlös- bzw. Verlustrechnung erfolgen. Die Auswertung erfolgte mit Microsoft Excel Version 2013 (Fa. Microsoft Corporation, Redmond, USA) und mittels SPSS Statistics, Version 24.0 (Fa. IBM, Armonk, New York, USA).

\section{Ergebnisse}

Im erfassten Zeitraum von 36 Monaten wurden insgesamt 19.374 Fälle in die Analyse einbezogen, die sich in der Subgruppenanalyse wie folgt darstellen:

Risikogruppe 1. Primäre Fehlbelegungen wurden 2017 in 115 Fällen dokumentiert, 2018 in 106 Fällen und 2019 in 24 Fällen. Das mittlere Alter betrug 43,7 $\pm 19,6$ Jahre (62\% weiblich). Der PCCL wies in $92 \%$ der Fälle den Wert 0 auf, (PCCL 1: $5 \%$, PCCL 2: $3 \%$ ). $47 \%$ entstammen der UCh, $27 \%$ der WCh, $11 \%$ der PCh, $8 \%$ der ACh, $7 \%$ der ORT. 


\begin{tabular}{|c|c|c|c|c|c|c|}
\hline DRG & $\begin{array}{l}\text { Risikogruppe } 1 \\
\text { Fehlbelegung }\end{array}$ & $\begin{array}{l}\text { Risikogruppe } 2 \\
\text { AOP } 1 \text { und } 2\end{array}$ & $\begin{array}{l}\text { Risikogruppe } 3 \\
\text { Eintagesfälle }\end{array}$ & $\begin{array}{l}\text { Schnittmenge } \\
\text { Gruppe } 1 \text { und } 2\end{array}$ & $\begin{array}{l}\text { Fallzahl } \\
\text { gesamt }\end{array}$ & $\%$ \\
\hline I23B & 57 & 148 & 166 & 95 & 276 & $17,0 \%$ \\
\hline I32G & 21 & 44 & 55 & 26 & 94 & $5,8 \%$ \\
\hline I18B & 3 & 34 & 61 & 16 & 82 & $5,0 \%$ \\
\hline $124 Z$ & 9 & 31 & 57 & 25 & 72 & $4,4 \%$ \\
\hline I27D & 8 & 43 & 25 & 10 & 66 & $4,1 \%$ \\
\hline I32F & 5 & 46 & 10 & 8 & 53 & $3,3 \%$ \\
\hline $\mathrm{I} 2 \mathrm{OH}$ & 5 & 28 & 32 & 13 & 52 & $3,2 \%$ \\
\hline $159 Z$ & 1 & 25 & 31 & 9 & 48 & $3,0 \%$ \\
\hline I18A & 1 & 34 & 13 & 3 & 45 & $2,8 \%$ \\
\hline J11B & 3 & 33 & 22 & 15 & 43 & $2,6 \%$ \\
\hline sonstige & & & & & 795 & $48,8 \%$ \\
\hline Summe & & & & & 1626 & $100,0 \%$ \\
\hline
\end{tabular}

Abb. $1 \varangle$ Verteilung der zehn häufigsten DRG nach Risikogruppen
Risikogruppe 2. Im Jahr 217 erfolgten bei 284 Fällen ausschließlich Prozeduren der Kategorie AOP 1 und/oder 2, in 2018 bei 257 Fällen und in 2019 bei 223 Fällen mit einem mittleren Alter von 44,4 \pm 18,8 Jahren (63\% männlich). Der PCCL betrug in $90 \%$ den Wert 0 (PCCL 1: $5 \%$, PCCL 2: $3 \%$, PCCL 3: $2 \%)$. Die meisten Fälle entstammen der UCh (51\%), gefolgt von der ACh (22\%), PCh (19\%), ORT (6\%) und WCh (2\%). Eine Operation erfolgte am häufigsten aufgrund der Hauptdiagnosegruppe S83 (Luxation, Verstauchung oder Zerrung des Kniegelenks und von Bändern des Kniegelenks, $11 \%$ der Fälle), nachfolgend aufgrund S82 (Unterschenkelfraktur, $9 \%)$, M23 (Binnenschädigung des Kniegelenks, 6\%) S52 (Fraktur des Unterarms, $6 \%$ ) und S42 (Schulter- und Oberarmfraktur, $4 \%)$.

Risikogruppe 3. Für die hypothetische Risikogruppe an elektiven Eintagesfällen ergibt sich im Jahr 2017 eine Fallzahl von 281, 2018 von 327 und 2019 von 283 mit einem mittleren Alter von 46,4 $\pm 16,6$ Jahren (53\% männlich). In über $91 \%$ betrug der PCCL den Wert 0 (PCCL 1: $2 \%$, PCCL 2: $4 \%$ und PCCL 3: 2\%). $44 \%$ der Fälle entstammen der UCh, gefolgt von der ACh (30\%), PCh (15\%), ORT (10\%) und WCh (1\%). Die häufigste Hauptdiagnosegruppe war S52 (18\%), gefolgt von M23 (10\%), S82 (8\%), S42 (6\%) und S83 (4\%).

Schnittmenge. Die Risikogruppen 2 und 3 weisen eine Schnittmenge von 98 Fällen bzw. 51,60 CM-Punkten in 2017, 103 Fällen bzw. 54,58 CM-Punkten in 2018 und
73 Fällen bzw. 40,30 CM-Punkten in 2019 auf.

Die Verteilung der DRG nach Risikogruppe zeigt Abb. 1 (exklusive der Schnittmengenfälle). Die 10 häufigsten Fallpauschalen bilden 51,1\% der Risikofälle $a b$.

\section{Ökonomische Betrachtung}

Die Verteilung der CM-Punkte ist in - Abb. 2 dargestellt. Die Summe von Fallzahl, CM und Belegtagen pro Jahr ergibt sich durch Addition der jeweiligen Werte abzüglich der Schnittmenge der Risikogruppen 2 und 3. Durch Multiplikation der jeweiligen LBFW ergeben sich aus dem CM die gefährdeten Erlössummen der 3 Jahre. Das ökonomische Risiko der 3 betrachteten Gruppen ist in $\mathbf{0}$ Tab. 1 zusammengefasst. Den Erlösverlusten aus der Ambulantisierung von operativen Eingriffen stehen die (fiktiven) Erlöse für das ambulante Operieren gegenüber. Diese betrugen für das Jahr 2017 im Mittel und inklusive einer Sachkostenerstattung 394,65€, für 2018 415,42€ und für $2019321,30 €$. Würden alle Risikofälle ambulant operiert, lägen die ambulanten Erlöse dafür 2017 bei $229.684 €, 2018$ bei $243.853 €$ und 2019 bei $146.836 €$. Das verbleibende Erlösrisiko würde dabei für 2017 1.049.207€, für 2018 1.076.727€ und für $2019923.163 €$ betragen.

\section{Diskussion}

\section{MDK-Reformgesetz zur Ambulantisierung}

Der gesetzgeberische Wille zu wirtschaftlicherem Agieren im Gesundheitswesen soll u.a. durch das MDK-Reformgesetz und die Verlagerung bislang stationär erbrachter Leistungen in den ambulanten Versorgungsektor bzw. die Versorgung nach § 115b SGB V umgesetzt werden [6]. In Deutschland ist die Anzahl ambulant durchgeführter Operationen im Krankenhaus seit dem Jahr 2008 bis 2017 um ca. $12,1 \%$ gestiegen, aber weniger stark als die Anzahl vollstationärer Operationen (um ca. 23,3\%) [12]. Die Frage nach der Notwendigkeit und Dauer von Krankenhausbehandlungen führt immer wieder zu Diskussionen zwischen Leistungserbringern und Kostenträgern. Ursächlich ist grundlegend die Diskussion über die Bindung von Ressourcen, welche an anderer Stelle besser eingesetzt werden könnten.

Die Gesundheitsversorgung soll nach $\S 12$ des SGB V patientenorientiert so gestaltet werden, wie es individuell medizinisch, qualitativ aber auch ökonomisch sinnvoll ist [5]. Leider ist häufig festzustellen, dass das Bestreben der Kostenträger häufig von einer ökonomischen Maxime geprägt ist. Für Krankenhäuser gab es bislang wenige Anreize für eine Verlagerung stationärer Operationen in die ambulante Versorgung, nicht zuletzt, weil die stationäre Versorgung gegenüber der ambulanten deutlich besser vergütet ist. Weiterhin fehlen am Krankenhaus häufig effiziente 


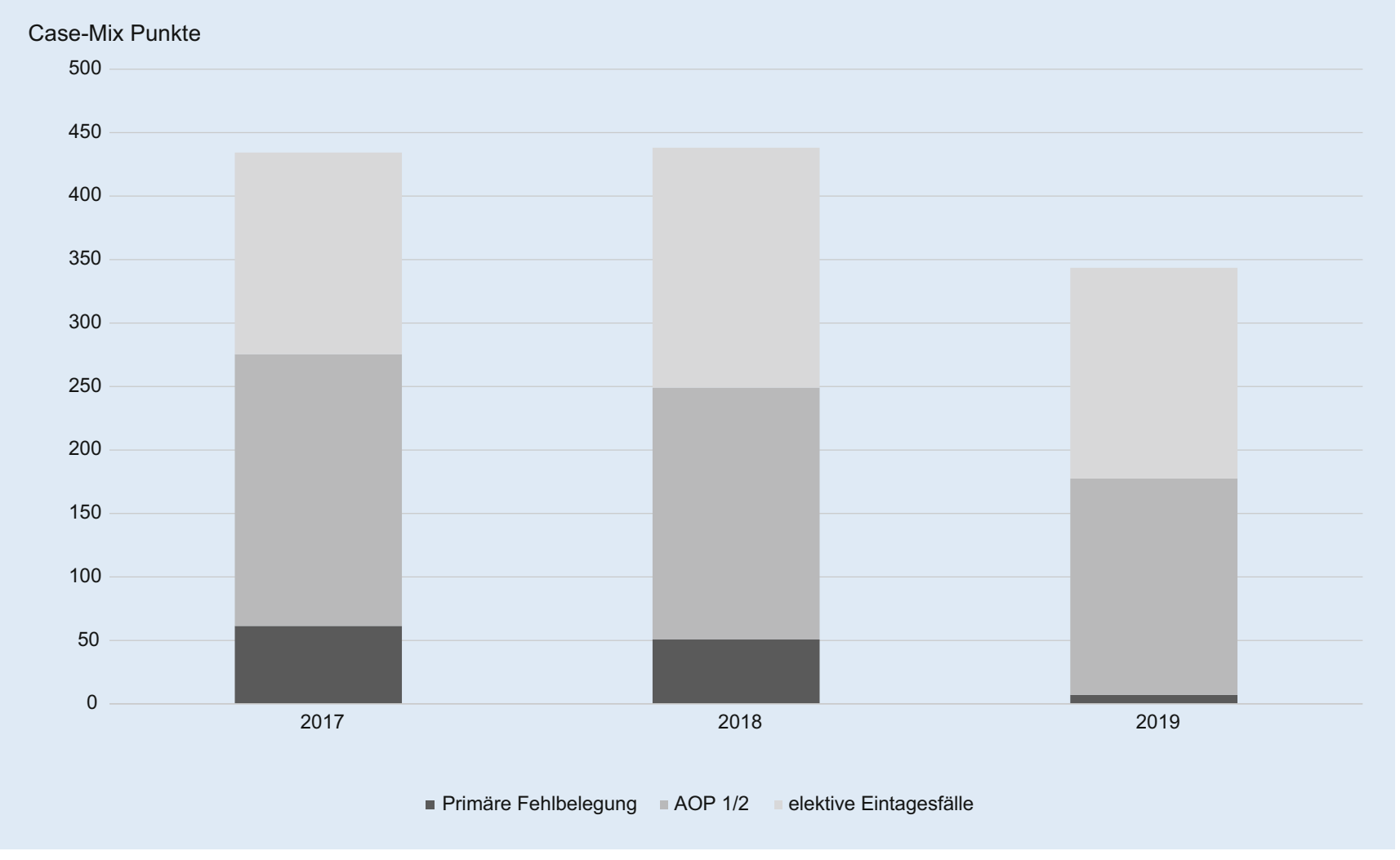

Abb. 2 A Case-Mix der 3 Gruppen mit Ambulantisierungsrisiko 2017-2019

Parallelstrukturen für das ambulante Operieren.

\section{Ambulantisierungsrisiko stationärer Fälle}

Ziel dieser Arbeit ist die Bewertung des Ambulantisierungsrisikos stationärer Fälle eines universitären Maximalversorgers für Orthopädie und Unfallchirurgie anhand von 3 Patientengruppen. Bei Gruppe 1 ist das Risiko bereits eingetreten, da diese Fälle nach MDK-Prüfung und Leistungsentscheidung der Krankenkassen bereits einen sog. Fallartwechsel von stationär nach ambulant erfuhren. Aus der Fallübersicht geht hervor, dass häufig DRG der konservativen Wirbelsäulenbehandlung betroffen sind (I68D und I68E, 25\%). Hierbei ist nicht immer von einem realen Transferpotenzial auszugehen, da diese Patienten häufig in akuten Schmerzsituationen eine stationäre Behandlung benötigen. Diesen Patienten ist einem Fallartwechsel durch gewissenhafte Dokumentation zu begegnen. Allerdings fallen auch Patienten, die zu diagnostischen Zwecken bei Wirbelsäulenerkran- kungen stationär behandelt werden (z. B. Stufendiagnostik) in die Basis-DRG 168. Insbesondere hier gilt es, die Möglichkeit ambulanter Leistungserbringung zu prüfen.

Auch bei Behandlungsfällen der DRG I23B (Entfernung von Osteosynthesematerial) wird häufig keine stationäre Behandlungsnotwendigkeit gesehen. Diese sollte in der Tat individuell abgewogen werden, und ein Fallartwechsel ist wiederum nur mit einer entsprechenden Dokumentation und Begründung zu verhindern. Selbstredend sei an dieser Stelle auf wahrheitsgemäße Dokumentation hingewiesen, die aber vollständig sein muss, will man damit bei der MD-Begutachtung oder vor dem Sozialgericht Bestand haben. Jedoch zeigen bereits mehrere Studien, dass bereits die initiale operative Versorgung in einem ambulanten Setting durchgeführt werden kann $[17,20]$.

Gruppe 2 berücksichtigt Fälle, deren stationäre Operationen einem Eingriff der AOP-Kategorie 1/2 entsprechen. Wiederum stellt die DRG $123 \mathrm{~B}$ die größte Untergruppe dar. Die zugehörigen Hauptdiagnosen sind heterogen, aber größten- teils aus dem Formenkreis von Verletzungen der unteren Extremität (ca. 26\%). Hierbei ist zu berücksichtigen, dass bei Materialentfernungen der Frakturkode die Hauptdiagnose darstellt. Das Ambulantisierungsrisiko ist erneut individuell hoch einzuordnen.

Die Auswahl der Parameter der Gruppe 3 soll den komplikationslosen Eintagesfall beschreiben, deren Charakterisierung durch eine elektive Aufnahme andere Eintagesfälle wie z. B. zur Überwachung nach Schädel-Hirn-Trauma abgrenzt. Die Entlassart "normal“ grenzt Verstorbene ab. $\mathrm{Zu}$ den häufigsten Hauptdiagnosen zählen Frakturen des Unterarms, größtenteils distale Radiusfrakturen. Sofern keine offenen Frakturen, große Weichteilschäden oder sonstige komplizierende Umstände vorliegen, erfolgt die Versorgung größtenteils nicht akut [11]. Whiting et al. konnten zeigen, dass eine ambulante Operation geschlossener Frakturen der stationären Behandlung überlegen ist [23]. Hier besteht ein hohes Ambulantisierungsrisiko. Neben der Entfernung von Osteosynthesematerial (I23B) weisen zudem DRG mit Bezug zu arthroskopischen Eingriffen am 
Tab. 1 Case-Mix (CM), Belegtage und Erlösrisiko

\begin{tabular}{|c|c|c|c|c|c|c|c|c|c|}
\hline Entlassungsjahr & \multicolumn{3}{|c|}{2017} & \multicolumn{3}{|l|}{2018} & \multicolumn{3}{|l|}{2019} \\
\hline Risikogruppe & Fälle & CM & Belegtage & Fälle & CM & Belegtage & Fälle & CM & Belegtage \\
\hline 1: Primäre Fehlbelegung & 115 & 61,43 & 187 & 106 & 50,72 & 121 & 24 & 7,09 & 37 \\
\hline $2: A O P 1 / 2$ & 284 & 214,04 & 695 & 257 & 198,46 & 673 & 223 & 170,37 & 516 \\
\hline 3: elektive Eintagesfälle & 281 & 158,85 & 281 & 327 & 188,96 & 327 & 283 & 166,07 & 283 \\
\hline ./. Schnittmenge Gr. $2 / 3$ & 98 & 51,60 & 98 & 103 & 54,58 & 103 & 73 & 40,30 & 73 \\
\hline$\Sigma$ & 582 & 382,71 & 1065 & 587 & 383,56 & 1018 & 457 & 303,23 & 762 \\
\hline Erlösrisiko & \multicolumn{3}{|c|}{$1.278 .891 €$} & \multicolumn{3}{|c|}{$1.320 .580 €$} & \multicolumn{3}{|c|}{$1.070 .000 €$} \\
\hline Mittlerer Verlust pro Fall & \multicolumn{3}{|c|}{$2197 €$} & \multicolumn{3}{|c|}{$2250 €$} & \multicolumn{3}{|c|}{$2341 €$} \\
\hline
\end{tabular}

Kniegelenk (I24Z) ein Ambulantisierungsrisiko auf. Schwappach et al. zeigten in ihrer Patientenbefragung, dass ambulante Operationen einem Patientenbedürfnis entsprechen [21]. Grundlegend ist eine arthroskopische Behandlung von Kreuzbandrupturen und Meniskusnähten bzw. -resektionen ambulant in gleicher Qualität möglich [16, 18, 19, 22].

Die meisten Fälle mit Transferpotenzial können den Organisationseinheiten UCh, ACh und PCh zugeordnet werden. Dabei stellt sich in allen 3 Gruppen am häufigsten die DRG I23B heraus (ca. 19-23\%). In der Regel handelt es sich dabei um elektive stationäre Aufenthalte für Operationen überwiegend an "kleinen Knochen“ wie Schlüsselbein, Unterarm oder am Sprunggelenk, da die aufwendigeren Materialentfernungen (Wirbelsäule und Oberschenkel) nicht in dieser DRG enthalten sind. Prinzipiell kann hier eine ambulante Versorgung erwogen werden. Kritisch ist jedoch anzumerken, dass es sich auch um Ausbildungsinhalte handelt und ein komplettes Streichen dieser Operationen aus dem stationären Portfolio die Ausbildung schwächt. Daher wäre entweder ein Teiltransfer oder die Einbindung von Ausbildungsassistenten in das ambulante Setting zu erwägen.

Insgesamt zeichnen sich die Gruppen durch ein junges Patientenkollektiv aus (knapp über 40 Jahre), welches in ca. $90 \%$ keine relevanten Nebendiagnosen (PCCL 0) aufweist und potenziell ambulantisierbar erscheint. Aus dem „Katalog ambulant durchführbarer Operationen und sonstiger stationsersetzender Eingriffe" kann natürlich nicht die Verpflichtung hergeleitet werden, die dort aufgeführten Eingriffe ausschließlich ambulant zu erbringen. Grundsätzlich entscheidet der verantwortliche Arzt individuell über
Art, Umfang und Setting des operativen Eingriffs mit Hinblick auf den Gesundheitszustand und eine angemessene Nachbetreuung im häuslichen Bereich. Als Begründungs- und Bewertungshilfe haben die Selbstverwaltungspartner dazu den sog. G-AEP-Katalog abgestimmt (German Appropriate Evaluation Protocol). Eine verpflichtende Grundlage für oder gegen eine stationäre Behandlung stellt dieser jedoch nicht dar, sondern gibt vielmehr lediglich Orientierungskriterien.

Von den 3 Gruppen spiegelt Gruppe 1 das bereits eingetretene Risiko der Ambulantisierung wider. Das maximale Risiko stellt den Wegfall der Erlöse aller 3 Gruppen dar. Demgegenüber sind die ambulanten Erlöse gering und fangen den potenziellen Erlösausfall in keinem Fall adäquat ab. Bezogen auf die untersuchte Klinik bedeutet der potenzielle Erlösverlust rund 3-4\% der DRG-Erlöse des jeweiligen Jahres.

\section{Perspektive ambulantisierbarer Operationen}

Typischerweise ist eine Unfallchirurgie der Maximalversorgung für die Behandlung von Schwerstverletzten zuständig und strukturell und personell dahingehend organisiert. Durch die Inanspruchnahme stationärer Kapazitäten für dringlichere Eingriffe entstehen mitunter unverhältnismäßig lange Wartezeiten für elektiverer Operationen. Eine zunehmende Integration ambulanter Operationen mit einer entsprechenden Erwartungshaltung ist eine große Herausforderung. Demgegenüber hat die synergistische und effiziente Nutzung bestehender Ressourcen auch für ambulante Operationseinheiten Potenzial und ist als vorteilhaft anzusehen. Nicht nur strukturelle Ressourcen wie z.B. Apotheken- oder Sterilisationseinheiten, sondern auch persönliche Fähigkeiten wie eine entsprechende Qualifizierung von Fachund Pflegepersonal können integriert werden. Die Vermischung dieser Ressourcen ist wiederum komplex, und nicht selten entstehen hierbei Interessenkonflikte.

Als ökonomischer Anreiz einer zunehmenden Ambulantisierung operativer Eingriffe wird der Ausschluss ambulanter Leistungen des AOP-Katalogs von MD-Prüfungen gesehen. Hinzu kommt die eingeführte Strafzahlung für jede beanstandete Krankenhausrechnung ab 2022 (mind. 300€ bzw. höchstens 10\% des durch den MD geminderten Abrechnungsbetrags) [4]. Die Steigerung ambulanter Eingriffe könnte hier Streitpotenzial mindern. Ein nicht von der Hand zu weisender Vorteil des ambulanten Operierens ist die direkte Sachkostenerstattung außerhalb des Sprechstundenund Praxisbedarfs und unter Einhaltung des Wirtschaftlichkeitsgebots, die in $\S 44$ des Bundesmantelvertrags-Ärzte (BMV-Ä) geregelt ist [14].

Generell sind für einen praktischen Falltransfer stationär nach ambulant diverse Herausforderungen personeller, räumlicher und struktureller Art zu berücksichtigen. Ein funktionierendes Netzwerk, Kooperationen und ein sicher geplantes $\mathrm{Ma}-$ nagement sind uneingeschränkte Voraussetzungen für eine funktionierende Integration des ambulanten Operierens in ein Krankenhaus. Eine Umsetzung der Fallsteuerung ist ebenso durch die Anbindung eines MVZ und ambulantes Operationszentrums möglich und wird zunehmend in Deutschland umgesetzt [13]. Die Integration und Überschneidung stationärer und ambulanter Prozesse wird effektiven Nutzen durch synergistische Ressourcennutzung bringen, wenn die Hürden ei- 
nes komplexen Managements überwunden werden können.

Limitationen: Methodenkritisch ist zu erwähnen, dass es sich bei der vorliegenden Arbeit um eine "Single-center"-Studie an einem Universitätsklinikum handelt. Das zugrunde liegende Patientenkollektiv und die daraus abgeleiteten Risikopotenziale sind daher nicht ohne Weiteres auf andere Krankenhäuser zu übertragen. Das Rechnungsjahr 2019 ist mit den vorliegenden Zahlen noch nicht vollständig bezüglich der MD-Gutachten bereinigt. Die Fallzahl der primären Fehlbelegungen liegt voraussichtlich in der Größenordnung der Vorjahre.

\section{Fazit für die Praxis}

- Mit dem MDK-Reformgesetz soll das Ambulantisierungspotenzial weiter gefördert werden.

- Vor allem elektive „kleinchirurgische“ Eingriffe wie die Entfernung von Osteosynthesematerial bieten ein Transferpotenzial zum ambulanten Eingriff.

- Kritisch anzumerken ist, dass es sich hier aber auch um Ausbildungsinhalte handelt. Zudem sind für einen praktischen Falltransfer stationär nach ambulant diverse Herausforderungen personeller, räumlicher und struktureller Art zu berücksichtigen.

- In jedem Fall muss vorab eine entsprechende Vorselektion des Patientenguts erfolgen.

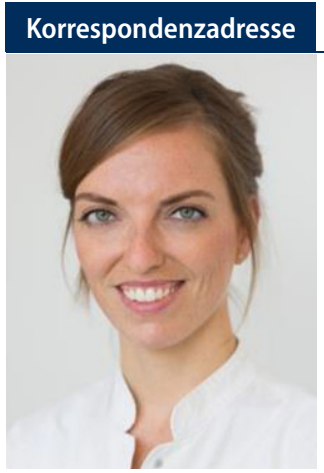

Dr. med. Dipl.-Ing. (BA) Jeanette Henkelmann, MHBA

Klinik und Poliklinik für Diagnostische und Interventionelle Radiologie, Universitätsklinikum Leipzig AöR

Liebigstraße 20, 04103 Leipzig, Deutschland jeanette.henkelmann@medizin.uni-leipzig.de
Funding. Open Access funding enabled and organized by Projekt DEAL.

\section{Einhaltung ethischer Richtlinien}

Interessenkonflikt. J. Henkelmann, R. Henkelmann und N. von Dercks geben an, dass kein Interessenkonflikt besteht.

Für diesen Beitrag wurden von den Autoren keine Studien an Menschen oder Tieren durchgeführt. Für die aufgeführten Studien gelten die jeweils dort angegebenen ethischen Richtlinien.

Open Access. Dieser Artikel wird unter der Creative Commons Namensnennung 4.0 International Lizenz veröffentlicht, welche die Nutzung, Vervielfältigung, Bearbeitung, Verbreitung und Wiedergabe in jeglichem Medium und Format erlaubt, sofern Sie den/die ursprünglichen Autor(en) und die Quelle ordnungsgemäß nennen, einen Link zur Creative Commons Lizenz beifügen und angeben, ob Änderungen vorgenommen wurden.

Die in diesem Artikel enthaltenen Bilder und sonstiges Drittmaterial unterliegen ebenfalls der genannten Creative Commons Lizenz, sofern sich aus der Abbildungslegende nichts anderes ergibt. Sofern das betreffende Material nicht unter der genannten Creative Commons Lizenz steht und die betreffende Handlung nicht nach gesetzlichen Vorschriften erlaubt ist, ist für die oben aufgeführten Weiterverwendungen des Materials die Einwilligung des jeweiligen Rechteinhabers einzuholen.

Weitere Details zur Lizenz entnehmen Sie bitte der Lizenzinformation auf http://creativecommons.org/ licenses/by/4.0/deed.de.

\section{Literatur}

1. Berger AK, Chopra S, Desai MM, Aron M, Gill IS (2016) Outpatient robotic radical prostatectomy: matched-pair comparison with inpatient surgery. J Endourol 30(Suppl 1):S52-S56. https://doi.org/ 10.1089/end.2016.0135

2. Bettin C, Nelson R, Rothberg D, Barg A, Lyman M, Saltzman C (2019) Cost comparison of surgically treated ankle fractures managed in an inpatient versus outpatient setting. J Am Acad Orthop Surg 27(3):e127-e134. https://doi.org/10.5435/ JAAOS-D-16-00897

3. Brenner G (1995) Die ambulante Chirurgie in Deutschland zwischen Bedarf und Wettbewerb. Chirurg 66(5):463-469

4. Bundesministerium der Justiz und für Verbraucherschutz (1988) Sozialgesetzbuch (SGB) Fünftes Buch (V) - Gesetzliche Krankenversicherung ( 275 Neuntes Kapitel, Medizinischer Dienst, Erster Abschnitt)

5. Bundesministerium der Justiz und für Verbraucherschutz (1988) Sozialgesetzbuch (SGB) Fünftes Buch (V) - Gesetzliche Krankenversicherung ( $\$ 12$ Wirtschaftlichkeitsgebot)

6. Bundesministerium der Justiz und für Verbraucherschutz (1988) Sozialgesetzbuch (SGB) Fünftes Buch (V) - Gesetzliche Krankenversicherung (§115b Ambulantes Operieren im Krankenhaus)

7. Bundesministerium der Justiz und für Verbraucherschutz (1988) Sozialgesetzbuch (SGB) Fünftes
Buch (V) - Gesetzliche Krankenversicherung (§ 39 Krankenhausbehandlung)

8. Deutscher Bundestag (2019) Entwurf eines Gesetzes für bessere und unabhängigere Prüfungen (MDK-Reformgesetz)

9. Deutscher Bundestag (2020) Gesetz zum Schutz der Bevölkerung bei einer epidemischen Lage von nationaler Tragweite

10. Frank C, Studier-Fischer S (2010) Ambulante Chirurgie/stationsersetzende Leistungen. Trauma Berufskrankh 12(S3):262-269. https://doi.org/10. 1007/s10039-009-1587-x

11. Geyer T, Hefele K, Gülke J, Gebhard F, Mentzel M (2011) Frühergebnisse nach palmarer winkelstabiler (multiaxialer) Plattenosteosynthese bei der Behandlung distaler Radiusfrakturen. Unfallchirurg 114(10):901-912. https://doi.org/10.1007/ s00113-010-1753-4

12. Kassenärztliche Bundesvereinigung (2018) Fachserie 12 Reihe 6.1.1 "Grunddaten der Krankenhäuser" Fachserie 12 Reihe 6.4 „Fallpauschalenbezogene Krankenhausstatistik". https:// gesundheitsdaten.kbv.de/cms/html/17031.php. Zugegriffen:27. Nov. 2020

13. Kassenärztliche Bundesvereinigung (2019) Medizinische Versorgungszentren Aktuell. Statistische Informationen Zum Stichtag 31.12.2019

14. Kassenärztliche Bundesvereinigung, Spitzenverband Bund der Krankenkassen (2021) Bundesmantelvertrag - Ärzte (BMV-Ä). § 44 Sonstige Abrechnungsregelungen

15. Lee DJ, Chin CJ, Hong CJ, Perera S, Witterick IJ (2018) Outpatient versus inpatient thyroidectomy: a systematic review and meta-analysis. Head Neck 40(1):192-202. https://doi.org/10.1002/hed. 24934

16. Lutz C, Baverel L, Colombet $P$, Cournapeau J, Dalmay F, Lefevre $\mathrm{N}$, Letartre $\mathrm{R}$, Potel J-F, Roussignol X, Cucurulo T, Servien E (2016) Pain after out-patient vs. in-patient $A C L$ reconstruction French prospective study of 1076 patients. Orthop Traumatol Surg Res 102(8S):S265-S270. https:// doi.org/10.1016/j.otsr.2016.08.009

17. Malik AT, Quatman CE, Khan SN, Phieffer LS, Rao P, Ly TV (2020) Outpatient versus inpatient surgical fixation of isolated ankle fractures: an analysis of 90-day complications, readmissions, and costs. J Foot Ankle Surg 59(3):502-506. https://doi.org/ 10.1053/j.jas.2019.09.030

18. Mall NA, Chalmers PN, Moric M, Tanaka MJ, Cole BJ, Bach BR, Paletta GA (2014) Incidence and trends of anterior cruciate ligament reconstruction in the United States. Am J Sports Med 42(10):2363-2370. https://doi.org/10.1177/0363546514542796

19. Min CJ, Partan MJ, Koutsogiannis P, Iturriaga CR, Katsigiorgis G, Cohn RM (2020) Risk factors for hospital admission in patients undergoing outpatient anterior cruciate ligament reconstruction: a national database study. J Orthop 22:436-441. https://doi.org/10.1016/j.jor.2020.09.020

20. Moon AS, McGee AS, Patel HA, Cone R, McGwin G, Naranje S, Shah A (2019) A safety and cost analysis of outpatient versus inpatient hindfoot fusion surgery. Foot Ankle Spec 12(4):336-344. https:// doi.org/10.1177/1938640018803699

21. Schwappach DLB, Strasmann TJ (2006) Ambulantes Operieren: Praxis oder Krankenhaus? Eine empirische Analyse von Bevölkerungspräferenzen für die Wahl des Behandlungssettings. Chirurg 77(2):166-172. https://doi.org/10.1007/s00104005-1102-2

22. Sochacki KR, Varshneya K, Calcei JG, Safran MR, Abrams GD, Donahue J, Sherman SL (2020) Comparing meniscectomy and meniscal repair: a matched 
cohort analysis utilizing a national insurance database. Am J Sports Med 48(10):2353-2359. https://doi.org/10.1177/0363546520935453

23. Whiting PS, Rice CD, Avilucea FR, Bulka CM, Shen MS, Obremskey WT, Sethi MK (2017) Patients at increased risk of major adverse events following operative treatment of distal radius fractures: inpatient versus outpatient. J Wrist Surg 6(3):220-226. https://doi.org/10.1055/s-00371599216

\section{Potential of inpatient cases of a university hospital for orthopedics and trauma surgery for outpatient care}

Background: The act of the Medical Service of the Health Funds (MDK) is intended to shift health services previously provided in inpatient care to the outpatient care sector in accordance with $\S 115 \mathrm{~b} \mathrm{SGB} \mathrm{V}$. The aim of this study was the investigation of potential groups of inpatient cases of a university hospital for trauma surgery and orthopedics, which might be at risk for transfer to surgical outpatient treatment. Methods: Data collection using SAP Data Warehouse included all inpatient cases 2017-2019, with subgroup analysis of economic parameters of three risk groups (RG): 1) primary misallocation, 2) procedures of AOP categories 1 and/or 2 and 3) elective 1-day cases. Furthermore, an analysis of epidemiological parameters and an economic evaluation were performed.

Results: Primary misallocations related to 245 cases, RG 2 had 764 cases and RG 3 had 891 cases. The average age was $45.5 \pm 17.7$ years and in $90 \%$ there were no relevant comorbidities (PCCL 0 ). The majority of cases were assigned to DRG I23B and I21Z (removal of osteosynthesis material, 15-23\%), followed by open or arthroscopic surgery of the extremities (DRG I32F, I32G, I24Z, I18B, 6-9\%). In cases of a statutory shift of inpatient to outpatient surgical procedures, the potential loss of revenue in 2017 was $€ 1,049,207$, in $2018 € 1,076,727$ and in $2019 € 923,163$.

Conclusion: Individual groups have an increased potential in certain DRGs for a shift from inpatient to outpatient surgical procedures and are at risk for relevant revenue reductions in the course of further expansion of outpatient care. Proactive patient management in terms of outpatient versus inpatient treatment and special management of staff and spatial resources are necessary to anticipate potential downstream revenue cuts.

\section{Keywords}

Outpatient surgery $\cdot$ DRG $\cdot$ Medical coding $\cdot$ Medical audit $\cdot$ Billing 
Hier steht eine Anzeige.

黑 Springer 